\title{
Neutralizing Antibody Evasion Ability of Adenovirus Vector Induced by the Bioconjugation of Methoxypolyethylene Glycol Succinimidyl Propionate (MPEG-SPA)
}

\author{
Yusuke Eto, ${ }^{a}$ Jian-Qing GaO, ${ }^{a, b}$ Fumiko Sekiguchi,${ }^{a}$ Shinnosuke Kurachi,${ }^{a}$ Kazufumi Katayama, ${ }^{a}$ \\ Hiroyuki Mizuguchi, ${ }^{c}$ Takao Hayakawa, ${ }^{d}$ Yasuo Tsutsumi,${ }^{a}$ Tadanori Mayumi, ${ }^{a}$ and \\ Shinsaku NAKAGAWA $*, a$ \\ a Graduate School of Pharmaceutical Sciences, Osaka University; 1-6 Yamadaoka, Suita, Osaka 565-0871, Japan: \\ ${ }^{b}$ Department of Pharmaceutics, College of Pharmaceutical Sciences, Zhejiang University; 353 Yanan Road, Hangzhou, \\ Zhejiang 310031, P.R. China: ${ }^{c}$ Division of Cellular and Gene Therapy Products, National Institute of Health Sciences; \\ and ${ }^{d}$ National Institute of Health Sciences; Tokyo 158-8531, Japan. Received March 3, 2004; accepted March 18, 2004
}

\begin{abstract}
Although adenovirus vectors (Ad) which possesses high transduction efficiency are widely used for gene therapy in animal models, clinical use is very limited. One of the main reason is that nearly $80 \%$ of human beings possess anti-Ad antibodies. In this study, we tried to modify Ad with methoxypolyethylene glycol (MPEG) activated by succinimidyl propionate, and, the neutralizing antibody evasion ability of PEGylated Ad was evaluated. The results demonstrated that PEG-Ad showed stronger protection ability against anti-Ad neutralizing antibody compared to that with unmodified-Ad. Considering there are many people carrying neutralizing antibody against Ad and readministration of Ad was necessary for treating chronic diseases, this strategy, which was also applicable to other vectors, can be used for developing improved vectors.
\end{abstract}

Key words adenovirus vector; polyethylene glycol; antibody; gene therapy

Although, the gene therapy for cancer or an incurable disease has attracted considerable attention, clinical use is very limited by the problem of vectors for transgenics. Vectors based on human adenoviruses have been developed as efficient vehicles for therapeutic transgenes in a wide variety of animal models. ${ }^{1-3)}$ Gene manipulated adenovirus vectors (Ad) have commanded considerable attention as gene delivery carriers since high-titer replication-deficient recombinant viral preparations can be easily generated, the viruses can be engineered to accommodate large DNA inserts, and they can show high gene expression in a wide variety of dividing and nondividing cells. ${ }^{4,5}$ However, first-generation adenoviruses possess a fundamental problem that both cellular and humoral immune responses prohibit gene transfer on readministration of Ad due to neutralization of viral particles by antibodies produced against capsid proteins. ${ }^{6,7)}$ Furthermore, many people carry immunity to Ad and a large amount of Ad administration causes side effects. Therefore, clinical application of Ad was very limited. ${ }^{8-10)}$ PEGylation, the covalent attachment of activated polyethylene glycol (PEG) to free lysine groups on Ad surface, is a promising strategy for overcoming these limitations. PEG-modification is a well-established technique for the modification of therapeutic peptides and proteins, and the validity of PEGylation has been reported by us and some other groups. ${ }^{11,12)}$ This method enabled transgenics in the presence of neutralizing antibodies of Ad without the necessity of recombining a gene in a vector like a gutless vector. ${ }^{13,14)}$ In this study, we used monomethoxypolyethylene glycol (MPEG) activated by succinimidyl propionate, which reacts preferentially with the $\varepsilon$-amino terminal of lysine residues, to covalently attached to the surface of Ad. And, we assessed the effects of biochemical modification of viral capsids with functionalized PEG on the neutralizing antibody against first-generation Ad.

\section{MATERIALS AND METHODS}

Cells and Animals HEK 293 cells, A549 human lung carcinoma cells were cultured with Dulbecco's Modified Eagle's Medium (DMEM) supplemented with $10 \%$ fetal calf serum (FCS). Female ICR mice were purchased from Nippon SLC (Kyoto, Japan) and used at 6 weeks-old stage.

Adenovirus ad expressing firefly Luciferase under the control of cytomegalovirus (CMV) promoters were amplified in 293 cells, using a modification of established methods and purified from cell lysates by banding twice on $\mathrm{CsCl}$ gradients, dialyzed and stored at $-80^{\circ} \mathrm{C}$. The Ad used in this study were constructed by an improved in vitro ligation method as described previously. ${ }^{15}$ Viral particle titer was spectrophotometrically determined by the established method. ${ }^{16)}$

PEGylation of Ad Activated methoxypolyethylene glycol succinimidyl propionate (MPEG-SPA, MW 5000, Shearwater Corporation) was used in this study (Fig. 1). Ad was reacted with 100 molar excess of mPEG-SPA for viral lysine residue at $37^{\circ} \mathrm{C}$ for $45 \mathrm{~min}$ with gentle stirring $(300 \mathrm{rpm})$. The particle size of PEG-Ad was measured by ZETASIZER 3000HS (Malvern, U.K.).

Sodium Dodecyl Sulfate-Polyacrylamide Gel Electrophoresis (SDS-PAGE) Analysis SDS-PAGE analysis was conducted for detecting the PEGylated viral proteins. In short, the unmodified-Ad or PEG-Ad suspensions were mixed with the same volume of 2XSDS protein gel loading solution (Quality Biological, Inc. U.S.A.) and 5\% (v/v) 2-

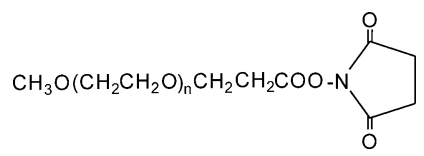

Fig. 1. mPEG-SPA Methoxypolyethylene Glycol Succinimidyl Propionate MW 5000. 
mercaptothanol was added to the final solution. After heating at $95^{\circ} \mathrm{C}$ for $5 \mathrm{~min}$, the mixture was added to $4-20 \%$ of polyacrylamide gel (PAG Mini 4/20) and electrophoresis was carried out using SDS-PAGE buffer. Full range Rainbow ${ }^{\mathrm{TM}}$ molecular weight marker (Amersham Life Science, U.S.A.) was used as the marker in this study. The gel was stained for viral hexon using Coomassie blue. The bioconjugation ratio of PEGylated Ad was calculated by the colority of the hexon band and the PEGylated hexon using NIH Image software.

Preparation of Ad Antiserum Ad antiserum was obtained from ICR mouse according to the approach described previously. ${ }^{17,18)}$ In brief, female ICR mouse (6 weeks old) was administered hypodermically with a dose of $10^{10}$ viral particles of conventional Ad with Freund's complete adjuvant in $100 \mu \mathrm{l}$ of PBS. Another $10^{10}$ viral particles of conventional Ad were hypodermically administered with Freund's incomplete adjuvant after 2 and 4 weeks. Then serum of the mouse was collected after 1 week and filtered, and stored at $-20^{\circ} \mathrm{C}$.

Transduction Efficiency of PEG-Ad and UnmodifiedAd into A549 Cells in the Presence or Absence of Ad Antiserum A549 cells $\left(1 \times 10^{4}\right.$ cells $)$ were seeded into a 48 well plate with $500 \mu \mathrm{l}$ medium. On the following day, the cells were transduced with 1000 particles/cell of unmodifiedAd or PEG-Ad respectively in a final volume of $500 \mu \mathrm{l}$ in the presence of $0,42,125 \mathrm{ng}$ protein/well of Ad antiserum. After $24 \mathrm{~h}$ cultivation, luciferase activity was measured using Luciferase Assay System (Promega, U.S.A.) and Microlumat Plus LB 96 (Perkin Elmer, U.S.A.) after cells were lysed with Luciferase Cell Culture Lysis Reagent (Promega, U.S.A.) according to the manufacturer's instruction.

\section{RESULTS AND DISCUSSION}

In this study, we tried to modify Ad with MPEG activated by succinimidyl propionate which was safety and widely used. ${ }^{19)}$ A simple and practicable method for constructing of PEG-Ad was employed. After reaction, the sizes of PEG-Ad and unmodified-Ad were measured. The results showed that the particle size of Ad was increased with PEGylation. The average viral particle size of PEG-Ad was about $10 \mathrm{~nm}$ bigger than that observed in the unmodified-Ad (Table 1). And SDS-PAGE analysis showed the presence of a new band of PEGylated viral capsid protein, hexon (Fig. 2). We also demonstrated that all Ad were conjugated by activated PEG, and there was no unmodified Ad mixed with the PEGylated ones (data not shown).

For evaluating the protection ability of PEG-Ad against the neutralizing antibodies, PEG-Ad and unmodified-Ad were added to A549 cells in the presence or absence of neutralizing antibodies against adenovirus capsid proteins, and the transduction levels were compared. Transduction efficiency of the unmodified-Ad was significantly reduced by the neutralizing antibodies (Fig. 3). In the presence of neutralizing antiserum of $42 \mathrm{ng}$, the luciferase gene expression of unmodified-Ad was less than half of that without antiserum, whereas PEG-Ad remained about $80 \%$ of its gene expression. In the presence of antibodies of $125 \mathrm{ng}$, the gene expression of unmodified-Ad was only $2.5 \%$ compared to that in the absence of antibodies, however, PEG-Ad showed more than 10 -fold of antibody evasion ability compared to unmodified-Ad. As anticipated, the absolute level of gene expres-
Table 1. Viral Particle Sizes of PEGylated Ad and Unmodified-Ad

\begin{tabular}{cc}
\hline \hline $\begin{array}{c}\text { Ratio } \\
(\text { Ad }: \text { PEG })^{a}\end{array}$ & $\begin{array}{c}\text { Vector size } \\
(\mathrm{nm})\end{array}$ \\
\hline $1: 0$ (unmodified) & $113.3 \pm 0.76$ \\
$1: 100($ PEG-Ad) & $123.8 \pm 0.98$ \\
\hline a) Amount of PEG to lysine residue of adenovirus vector capsid protein $(\mathrm{mol}: \mathrm{mol})$.
\end{tabular}

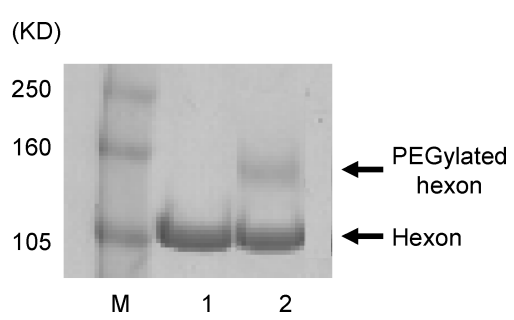

Fig. 2. SDS-PAGE Analysis of PEGylated Hexon

Lane M, protein marker; lane 1, unmodified-Ad; lane 2, PEG-Ad. Further details are described in Materials and Methods.

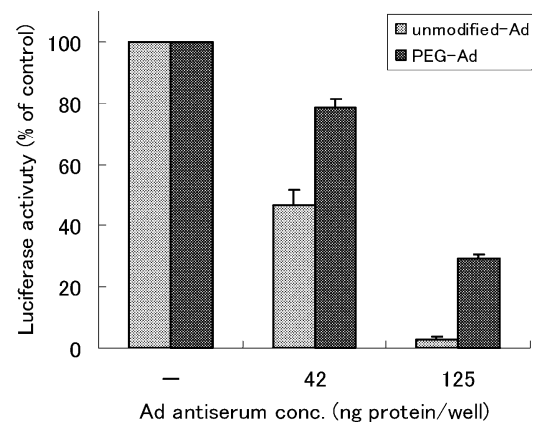

Fig. 3. Transduction Efficiency of Unmodified-Ad and PEG-Ad in the Presence or Absence of Ad Antiserum

A549 cells $\left(1 \times 10^{4}\right.$ cells $)$ were transduced with 1000 particles/cell of unmodified-Ad and PEG-Ad in the presence or absence of Ad antiserum respectively. Luciferase expression was measured after $24 \mathrm{~h}$. Each point was represented as mean \pm S.D. $(n=3)$.

sion of PEG-Ad was decreased to about 1/300 compared to that of unmodified-Ad in the absence of antibodies due to the effect that PEG chains prevented the interaction between $\mathrm{Ad}$ and coxsackie-adenovirus receptor (CAR). For developing novel Ad which possess a targeting ability, it is indispensable to block the route of Ad infection through CAR because of its broad expression in many tissues. So as the next step, we are trying to construct Ad which was conjugated by PEG with targeting molecule on the tip of the PEG. And we expect that this novel Ad will enhance the transduction efficiency of PEG-Ad at the same time keep the evading ability from the neutralizing antibodies.

One of the goals of Ad PEGylation is to provide a means to overcome the major limitation of Ad gene therapy, namely, antibody neutralization. The results presented here demonstrate that PEGylation can notably improve the ability of Ad to escape antibody neutralization in vitro by the steric hindrance of PEG chains. This antibody evasion ability is essential for clinical applications, because nearly $80 \%$ of human beings possess anti-Ad antibodies and readministration is indispensable in some cases like treating chronic diseases, such as cystic fibrosis and hemophilia. Furthermore, antibody evasion ability enabled decrease of the amount of medical re- 
quired to antibody carriers, and result in a reduction of side effects.

As next step, we are trying to evaluate its antibody evasion ability in vivo aiming for clinical use. Especially considering that the PEG-Ad used in this study was with low modification rate $(34 \%$, confirmed by SDS-PAGE, Fig. 2), we anticipated that highly modified PEG-Ad would protect it from neutralizing antibody more efficiently via stronger steric hindrance of PEG chains. Including of the other merits possessed by PEG-Ad, such as the extension of blood retention half-time and reduction of antigenicity. These approaches which are applicable to other vectors and other high compounds will promote development of the novel intelligent virus vectors.

Acknowledgements This study was supported in part by the Research on Health Sciences focusing on Drug Innovation from The Japan Health Sciences Foundation; by Grantsin-Aid for Exploratory Research from the Ministry of Education, Culture, Sports, Science and Technology of Japan; and by grants from the Ministry of Health and Welfare in Japan.

Y. Eto and J. Gao contributed equally to the work.

\section{REFERENCES}

1) Gao J. Q., Tsuda Y., Katayama K., Nakayama T., Hatanaka Y., Tani Y., Mizuguchi H., Hayakawa T., Yoshie O., Tsutsumi Y., Mayumi T., Nakagawa S., Cancer Res., 63, 4420-4425 (2003).

2) Crystal, R. G., Science, 270, $404-410$ (1995).
3) Wilson J. M., N. Engl. J. Med., 334, 1185-1187 (1996).

4) Smith T. A., Mehaffey M. G., Kayda D. B., Saunders J. M., Yei S., Trapnell B. C., McClelland A., Kaleko M., Nat. Genet., 5, 397-402 (1993).

5) Hitt M., Addison C., Graham F., Adv. Pharmacol., 40, 137-206 (1997).

6) Connelly S., Andrews J. L., Gallo A. M., Kayda D. B., Qian J., Hoyer L., Kadan M. J., Gorziglia M. I., Trapnell B. C., McClelland A., Kaleko M., Blood, 91, 3273-3281 (1998).

7) Zimmer K. P., Bendiks M., Mori M., Kominami E., Robinson M. B., Ye X., Wilson J. M., Mol. Med., 5, 244-253 (1999).

8) Wohlfart C., J. Virol., 62, 2321-2328 (1988).

9) Mastrangeli A., Harvey B. G., Yao J., Wolff G., Kovesdi I., Crystal R. G., Falck-Pedersen E., Hum. Gene Ther, 7, 79 -87 (1996).

10) Yei S., Mittereder N., Tang K., O’Sullivan C., Trapnell B. C., Gene Ther., 1, 192-200 (1994).

11) Inada Y., Furukawa M., Sasaki H., Kodera Y., Hiroto M., Nishimura H., Matsushima A., Trends Biotechnol., 13, 86-91 (1995).

12) Tsutsumi Y., Tsunoda S., Kamada H., Kihira T., Nakagawa S., Kaneda Y., Kanamori T., Mayumi T., Br. J. Cancer, 74, 1090-1095 (1996).

13) Von Seggern D. J., Chiu C. Y., Fleck S. K., Stewart P. L., Nemerow G. R., J. Virol., 73, 1601-1608 (1999).

14) Koizumi N., Mizuguchi H., Sakurai F., Yamaguchi T., Watanabe Y., Hayakawa T., J. Virol., 77, 13062-13072 (2003).

15) Mizuguchi H., Kay M. A., Hum. Gene Ther, 9, $2577-2583$ (1998).

16) Maizel J. V., Jr., White D. O., Scharff M. D., Virology, 36, 115-125 (1968).

17) Li W. M., Mayer L. D., Bally M. B., J. Pharmacol. Exp. Ther, 300, 976-983 (2002)

18) Chillon M., Lee J. H., Fasbender A., Welsh M. J., Gene Ther., 5, $995-$ 1002 (1998).

19) Tsutsumi Y., Kihira T., Tsunoda S., Kamada H., Nakagawa S., Kaneda Y., Kanamori T., Mayumi T., J. Pharmacol. Exp. Ther, 278, 10061011 (1996). 